\title{
Experimental and Numerical Study of Thermal Effect of Catalysis on the Surfaces of Metals and Quartz in Underexpanded Jets of Dissociated Air
}

\author{
A.V. Chaplygin ${ }^{1}$, A.N. Gordeev ${ }^{1}$, A.F. Kolesnikov ${ }^{1}$, V.I. Sakharov ${ }^{2}$, \\ S.A. Vasil'evskii ${ }^{1}$ \\ ${ }^{1}$ Ishlinsky Institute for Problems in Mechanics, Russian Academy of Sciences, \\ Moscow, 119526, Russia \\ ${ }^{2}$ Institute of Mechanics, Moscow State University, Moscow, 119192, Russia \\ koles@ipmnet.ru, sakharov@imec.msu.ru
}

\begin{abstract}
The heat transfer from supersonic under-expanded jets of dissociated air to the surface of a 30$\mathrm{mm}$ diameter cylindrical water-cooled model with a rounded edge was experimentally studied by the RF-plasmatron IPG-4 at the RF-generator power of $64 \mathrm{~kW}$ and air mass flow rate of $3.6 \mathrm{~g} / \mathrm{s}$. Stagnation point heat fluxes were measured by flat calorimetric probes embedded in the nose part of the model. Data were obtained using probes made of different materials: gold $(\mathrm{Au})$, silver $(\mathrm{Ag})$, copper $(\mathrm{Cu})$, molybdenum $(\mathrm{Mo})$, beryllium $(\mathrm{Be})$, tantalum $(\mathrm{Ta})$, niobium $(\mathrm{Nb})$ and quartz.

For the same mode of the RF-plasmatron operation, numerical simulation of the underexpanded jet of chemically nonequilibrium air plasma around the cylindrical model in the framework of the Navier-Stokes and simplified Maxwell equations is performed. Stagnation point heat fluxes at the model are calculated depending on the effective recombination coefficient of atomic components $\gamma$ in the range $0 \leq \gamma \leq 1$. From the comparison of experimental and calculated heat fluxes, the values of $\gamma$ for the above materials are determined and the quantitative scale of the catalytic properties of the metals and quartz considered is established. Data on the catalytic properties of metals and quartz are compared with the results obtained earlier in subsonic jets of dissociated air.
\end{abstract}

Keywords: RF-plasmatron, dissociated air, under-expanded jet, heat transfer, catalytic recombination of atoms, catalytic properties of metals and quartz.

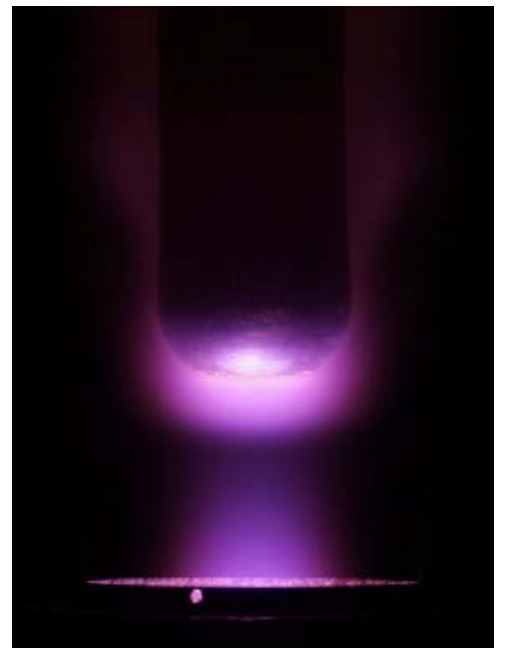

Flow pattern of supersonic air plasma flow around a water-cooled spherical blunt model with a flat nose 


\title{
Экспериментальное и численное исследование теплового эффекта катализа на поверхностях металлов и кварца в недорасширенных струях Диссоциированного воздуха
}

\author{
С.А. Васильевский ${ }^{1}$, А.Н. Гордеев ${ }^{1}$, А.Ф. Колесников ${ }^{1}$, В.И. Сахаров ${ }^{2}$, \\ А.В. Чаплыгин ${ }^{1}$ \\ ${ }^{1}$ ИПМех РАН, 119526, Москва, пр-т Вернадского, д. 101, корп. 1 \\ ${ }^{2}$ НИИ механики МГУ, 119192, Москва, Мичуринский проспект, д. 1 \\ koles@ipmnet.ru, sakharov@imec.msu.ru
}

\begin{abstract}
Аннотация
На ВЧ-плазмотроне ВГУ-4 экспериментально исследован теплообмен сверхзвуковых недорасширенных струй диссоциированного воздуха с поверхностью цилиндрической водоохлаждаемой модели диаметром 30 мм со скругленной кромкой и плоским притуплением носовой части при мощности ВЧ-генератора 64 кВт, расходе воздуха 3.6 г/с. Для измерения тепловых потоков использовались плоские калориметрические датчики, встроенные в носовую часть модели. Получены данные с помощью датчиков из различных материалов: золота $(\mathrm{Au})$, серебра $(\mathrm{Ag})$, меди $(\mathrm{Cu})$, молибдена (Мо), бериллия $(\mathrm{Be})$, тантала (Тa), ниобия $(\mathrm{Nb})$ и кварца.

Для этого же режима работы ВЧ-плазмотрона выполнено численное моделирование обтекания цилиндрической модели недорасширенной струей химически неравновесной воздушной плазмы в рамках уравнений Навье-Стокса и упрощенных уравнений Максвелла для высокочастотного электрического поля. Рассчитаны тепловые потоки в точке торможения модели в зависимости от эффективного коэффициента рекомбинации атомарных компонентов $\gamma$ в диапазоне $0 \leq \gamma \leq 1$. Из сравнения экспериментальных и рассчитанных тепловых потоков определены значения $\gamma$ для перечисленных выше материалов и установлена количественная шкала каталитичности рассмотренных металлов и кварца. Данные по каталитичности металлов и кварца сопоставлены с результатами, полученными ранее в дозвуковых струях диссоциированного воздуха.
\end{abstract}

Ключевые слова: ВЧ-плазмотрон, диссоциированный воздух, недорасширенная струя, теплообмен, каталитическая рекомбинация атомов, каталитические свойства металлов и кварца.

\section{1. Введение}

Вопросы численного и экспериментального исследования эффекта катализа на поверхностях различных материалов в диссоциированных потоках газов представляют большой интерес для задач моделирования процессов теплообмена спускаемых летательных аппаратов.

Индукционные плазмотроны являются удобным инструментом для постановки экспериментов в этой области.

Большинство работ, затрагивающих вопросы каталитических эффектов на поверхности материала, проводились для дозвуковых режимов обтекания моделей. Вопросы катализа в дозвуковых струях диссоциированного воздуха изучались на индукционных плазмотронах в ИПМех РАН [1, 2], ФГУП ЦНИИМаш [3], Институте гидродинамики фон Кармана 
[4]. В ЦАГИ каталитическая активность материала C-SiC определялась в режимах сверхзвукового обтекания образца потоками диссоциированного азота и воздуха [5].

В данной работе в продолжение [6] исследован тепловой эффект катализа на поверхностях ряда металлов и кварца в сверхзвуковой недорасширенной струе диссоциированного воздуха, который важен для ряда задач моделирования теплообмена.

\section{2. Эксперимент}

Эксперименты проводились на 100-киловаттном высокочастотном индукционном плазмотроне ВГУ-4. Принципиальная схема установки ВГУ-4 показана на рис. 1, a ее основные характеристики представлены в табл. 1. Подробное описание плазмотрона ВГУ-4 приведено в [7].



Рис. 1. Принципиальная схема высокочастотного индукционного плазмотрона ВГУ-4: 1 - ВЧ-генератор; 2 - блок согласования плазменной нагрузки с ВЧ-генератором; 3 - пульт управления; 4 - испытательная камера; 5 - водоохлаждаемый вакуумный трубопровод; 6 - теплообменник «плазма-вода»; 7 - вакуумные вентили; 8 - вакуумный трубопровод; 9 - вакуумный насос; 10 - вытяжной трубопровод; 11 - вытяжной вентилятор; 12 - компьютер системы сбора и регистрации данных; 13 - термовизор; 14 - пирометр; 15 - спектрометр

При проведении экспериментов используется компьютерная система сбора данных на основе АЦП E-270 (L-Card, 15 бит, 16 каналов, 100 Гц/канал) и программа обработки данных AC-Test.

Теплообмен в недорасширенных струях диссоциированного воздуха исследовался на медной водоохлаждаемой цилиндрической модели диаметром 30 мм со скругленной кромкой и плоским притуплением носовой части. Для измерений теплового потока в носовой части модели устанавливались проточные стационарные датчики (калориметры) с плоской тепловоспринимающей поверхностью из кварца высокой химической чистоты и различных металлов. Металлические тепловоспринимающие поверхности калориметров перед каждым экспериментом полировались и обрабатывались спиртом. Диаметр тепловоспринимающей поверхности калориметров составлял 13.8 мм.

Массовый расход охлаждающей воды в калориметрах измерялся ротаметром, калибровка которого выполнялась непосредственно перед экспериментом. Разность температур 
охлаждающей воды на выходе из калориметра и на входе в него измерялась экранированной дифференциальной термопарой хромель-алюмель.

Эксперименты по определению тепловых потоков в сверхзвуковых режимах плазмотрона ВГУ-4 проводились в недорасширенных струях высокоэнтальпийного воздуха, истекающих из водоохлаждаемого конического сопла с диаметром выходного сечения $D=50$ мм при давлении в затопленном пространстве 8.5 гПа, расходе воздуха 3.6 г/с и мощности ВЧ-генератора по анодному питанию 64 кВт.

Расстояние между передней критической точкой модели и срезом сопла составляло 30 мм. Картина обтекания модели сверхзвуковой недорасширенной струей воздушной плазмы в ходе эксперимента представлена на рис. 2.

\section{Таблиияа 1}

Основные характеристики установки ВГУ-4

\begin{tabular}{|l|c|}
\hline Мощность анодного питания, кВт & $12 \div 85$ \\
\hline Частота, МГц & 1.76 \\
\hline Диаметр разрядного канала, мм & 80 \\
\hline Расход воздуха, г/с & $2 \div 6$ \\
\hline Режим течения потока плазмы & $\begin{array}{c}\text { Дозвуковой и } \\
\text { сверхзвуковой }\end{array}$ \\
\hline Давление в испытательной камере, гПа & $6 \div 1000$ \\
\hline
\end{tabular}

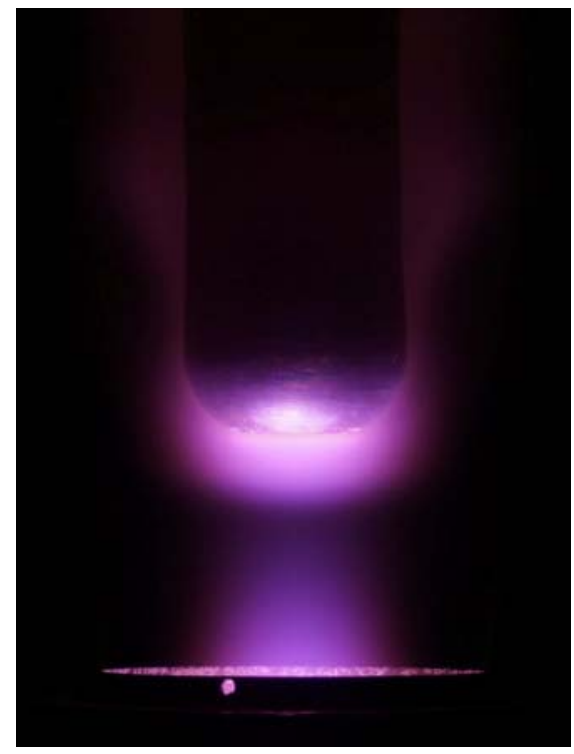

Рис. 2. Картина обтекания сверхзвуковым потоком воздушной плазмы водоохлаждаемой цилиндрической модели со сферическим затуплением и плоским носком

В экспериментах использовались датчики тепловых потоков с тепловоспринимающими поверхностями из различных материалов: медь $(\mathrm{Cu})$, серебро $(\mathrm{Ag})$, тантал (Та), молибден $(\mathrm{Mo})$, бериллий $(\mathrm{Be})$, ниобий $(\mathrm{Nb})$, золото $(\mathrm{Au})$ и кварц (три датчика с различной толщиной тепловоспринимающей стенки). Результаты экспериментов по измерению тепловых потоков к поверхностям металлов приведены в табл. 2. Результаты экспериментов по определению тепловых потоков к кварцевым датчикам с передними стенками различной толщины приведены в табл. 3 . 


\section{Таблица 2}

Тепловые потоки к датчикам из металлов

\begin{tabular}{|l|c|}
\hline $\begin{array}{c}\text { Материал тепловоспринимающей } \\
\text { поверхности датчика }\end{array}$ & $\begin{array}{c}\text { Измеренный } \\
\text { тепловой поток, } \\
\text { Вт/см }\end{array}$ \\
\hline Медь $(\mathrm{Cu})$ & 396 \\
\hline Серебро $(\mathrm{Ag})$ & 405 \\
\hline Ниобий $(\mathrm{Nb})$ & 255 \\
\hline Золото $(\mathrm{Au})$ & 252 \\
\hline Бериллий $(\mathrm{Be})$ & 230 \\
\hline Тантал $(\mathrm{Ta})$ & 224 \\
\hline Молибден $(\mathrm{Mo})$ & 197 \\
\hline
\end{tabular}

\section{Таблица 3}

Тепловые потоки к датчикам из кварца

\begin{tabular}{|c|c|c|c|}
\hline $\begin{array}{c}\text { № } \\
\text { Датчика }\end{array}$ & $\begin{array}{c}\text { Толщина } \\
\text { кварцевой стенки, } \\
\text { мм }\end{array}$ & $\begin{array}{c}\text { Рассчитанная } \\
\text { температура } \\
\text { поверхности } \\
\text { датчика, K }\end{array}$ & $\begin{array}{c}\text { Измеренный } \\
\text { тепловой } \\
\text { поток, Вт/см² }\end{array}$ \\
\hline 1 & 0.60 & 849 & 155 \\
\hline 2 & 0.81 & 956 & 141 \\
\hline 3 & 1.38 & 1203 & 123 \\
\hline
\end{tabular}

Наибольшие тепловые потоки зарегистрированы на датчиках из серебра и меди, причем в условиях проведенных экспериментов на этих датчиках получены практически одинаковые значения теплового потока.

Вторая группа материалов - золото, тантал, бериллий и ниобий. Эти материалы оказались близки по уровню зарегистрированных тепловых потоков. Реализуемые потоки для них ниже, чем у меди и серебра. Наименьший тепловой поток зарегистрирован на поверхности молибдена.

Минимальные тепловые потоки реализованы в исследовавшемся режиме на кварцевых датчиках. Отметим, что температура поверхности кварцевых датчиков и регистрируемые для одного и того же режима обтекания модели тепловые потоки зависят от толщины тепловоспринимающей стенки датчиков: чем больше толщина, тем меньше тепловой поток. Температура поверхности кварца определялась из решения одномерного уравнения теплопроводности в передней стенке датчика с учетом зависимости коэффициента теплопроводности кварца от температуры.

Результаты измерений давления торможения вдоль оси потока трубкой Пито и сравнение с расчетом для исследовавшегося режима приведены в [8].

\section{3. Численное моделирование}

Для численного моделирования течений в разрядном канале плазмотрона с индукционным нагревом газов и в истекающих недорасширенных струях диссоцированного и частично ионизованного воздуха применялась технология, основанная на комплексе программ численного интегрирования уравнений Навье-Стокса $[9,10]$ и специальных программ-генераторов, взаимодействующих с базами данных по термодинамическим и пере- 
носным свойствам индивидуальных газовых веществ [11]. Расчет переменного электромагнитного поля в разрядном канале плазмотрона проводился с использованием квазиодномерного приближения уравнений Максвелла для осредненной по времени комплексной амплитуды азимутальной составляющей электрического поля [12]. Энерговклад в плазму задавался исходя из значения КПД ВЧ-генератора 0.64.

Картина обтекания сферически затупленной модели с плоским носком изображена на рис. 3. Распределения энтальпии и массовых концентраций атомов $\mathrm{N}$ и О вдоль оси симметрии в этом течении приведены в [13].

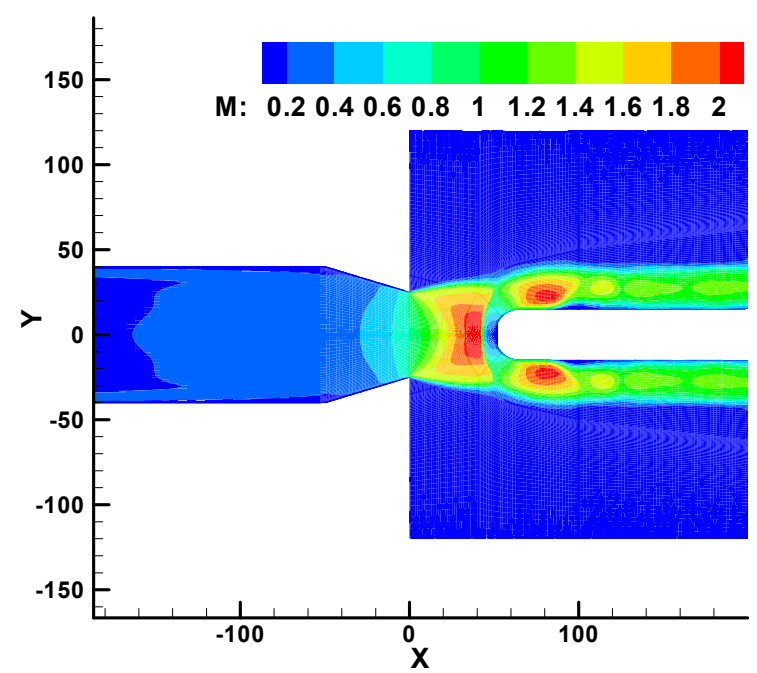

Рис. 3. Изомахи течения при обтекании недорасширенной струей воздуха сферической затупленной модели с плоским носком

В рассматриваемом случае для анализа теплообмена модели в сильно неравномерной недорасширенной струе необходимо учитывать распределение плотности теплового потока по поверхности датчика теплового потока. На рис. 4 приведены такие распределения $Q_{w}(R)$ для $\gamma=0$ и 1 при температурах стенки 300 и $1500 \mathrm{~K}$.

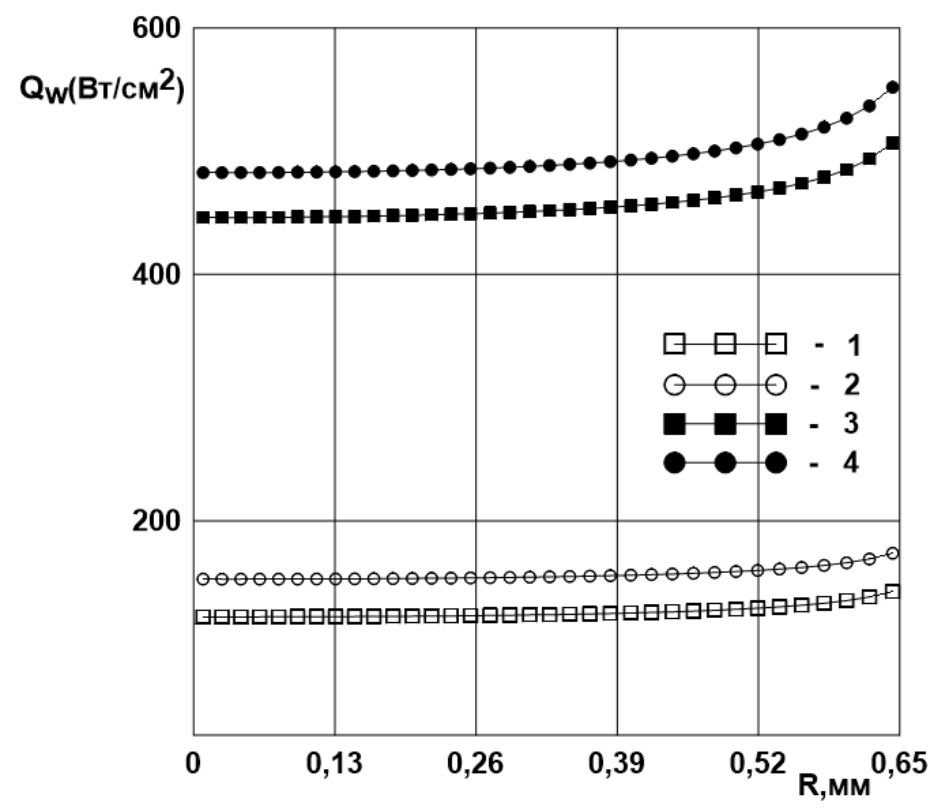

Рис. 4. Тепловые потоки по поверхности датчика: кривые 1, 2 соответствуют $\gamma=0$; кривые $3,4-\gamma=1$; кривые $1,3-T_{w}=1500 \mathrm{~K}$; кривые $2,4-T_{w}=300 \mathrm{~K}$ 
Для этих же значений $T_{w}$ и $\gamma$ на рис. 5 приведены распределения давления по радиусу калориметра. Как и ожидалось, профили давления практически не зависят от температуры стенки и коэффициента рекомбинации атомов.

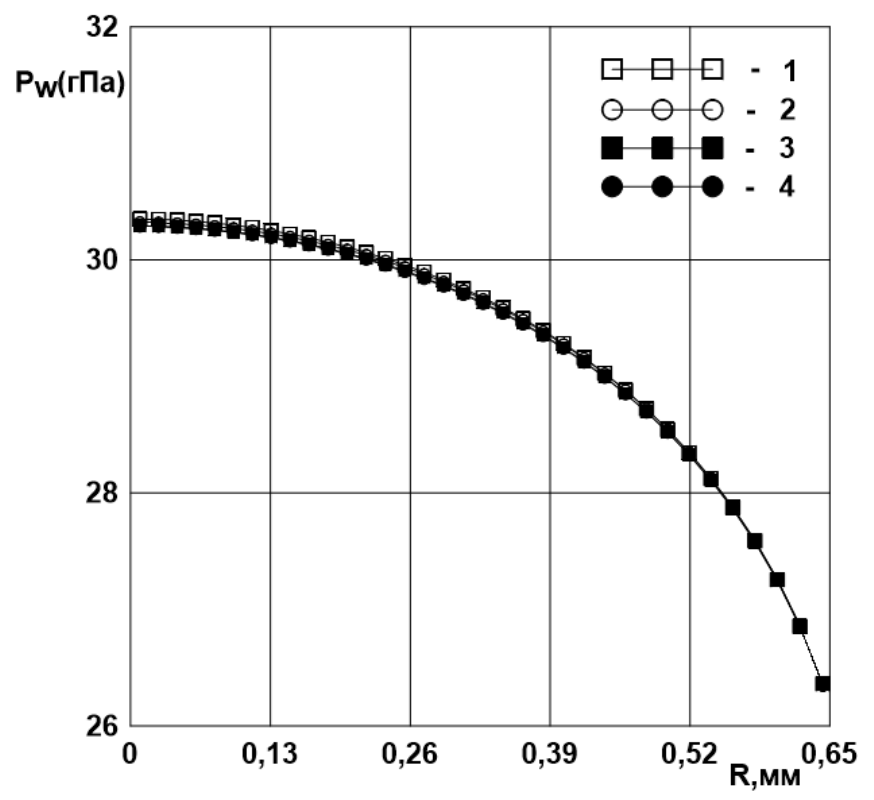

Рис. 5. Давление по поверхности датчика: кривые 1, 2 соответствуют $\gamma=0$; кривые $3,4-\gamma=1$; кривые 1,3 $-T_{w}=1500 \mathrm{~K} ;$ кривые $2,4-T_{w}=300 \mathrm{~K}$

Для корректного сопоставления рассчитанных и измеренных тепловых потоков необходимо использовать усредненные значения, полученные интегрированием профилей $Q_{w}(R)$ по радиусу калориметра. Вычисленные таким образом значения $q_{w}$ в зависимости от $T_{w}$ и $\gamma$ приведены в виде карты тепловых потоков на рис. 6.

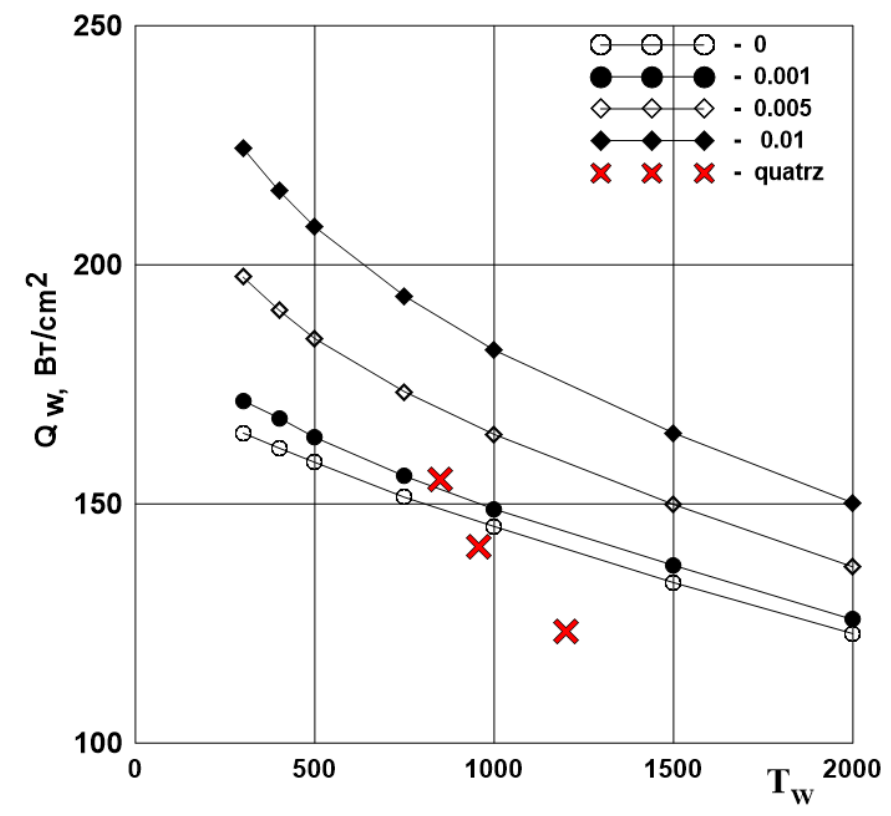

Рис. 6. Карта тепловых потоков и тепловые потоки к кварцевым датчикам различной толщины (0.6 мм, 0.81 мм и 1.38 мм) 
Для определения каталитических свойств водоохлаждаемых поверхностей кварцевыхдатчиков, поскольку температуры поверхности у них зависят от толщины тепловоспринимающей стенки $\left(T_{w}=849,956,1203 \mathrm{~K}\right)$, удобно воспользоваться рассчитанной картой тепловых потоков (усредненных): на рис. 6 кривые с точками соответствуют значениям $\gamma=0$, $0.001,0.005$ и 0.01 . В расчетное поле тепловых потоков попадает только одна экспериментальная точка, соответствующая минимальной толщине кварцевой стенки. Две другие расположены ниже расчетной кривой, соответствующей $\gamma=0$. Это объясняется тем, что экспериментально измеренные тепловые потоки занижены, поскольку не учтены тепловые потери вследствие растекания тепла в двух датчиках с относительно толстыми стенками. Рассчитанные температуры поверхности кварца для этих двух датчиков также занижены, поскольку определены из решения одномерного уравнения теплопроводности поперек тепловоспринимающей стенки. Максимальная погрешность измерения теплового потока имеет место для датчика с толщиной стенки 1.38 мм. Практическое определение значения $\gamma$ для кварца возможно с помощью кварцевого датчика с передней стенкой толщиной 0.6 мм.

Для определения коэффициента рекомбинации $\gamma$ на поверхности водоохлаждаемых металлических датчиков $\left(T_{w}=300 \mathrm{~K}\right)$ можно воспользоваться рис. 7 , где приведены расчетная зависимость $Q_{w}(\gamma)$ и экспериментальные данные по тепловым потокам для металлов.

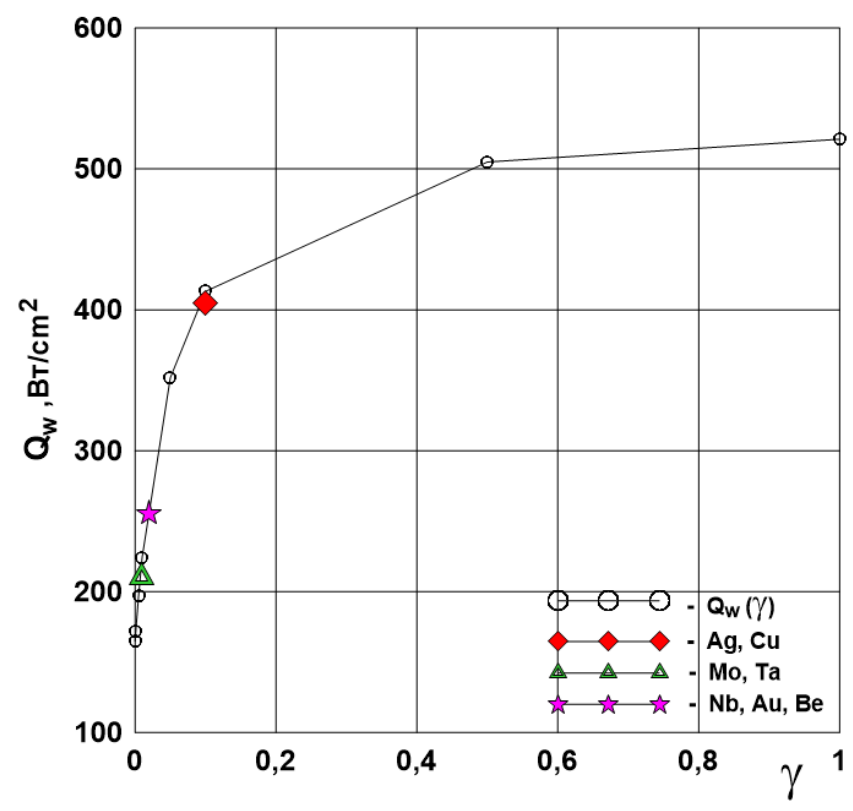

Рис. 7. Расчетная зависимость теплового потока к водоохлаждаемой поверхности модели в критической точке $\left(T_{w}=300 \mathrm{~K}\right)$ от величины $\gamma$ и экспериментальные данные; цветные маркеры соответствуют датчикам из различных металлов

Наибольшей каталитичностью обладают поверхности серебра и меди, причем значения $\gamma$ для этих металлов практически одинаковы.

Вторая группа материалов - золото, бериллий и ниобий. Эти материалы можно отнести к среднекаталитичным. Наименьшая каталитичность среди металлов отмечена у молибдена и тантала. Кварц также может быть отнесен к низкокаталитичным материалам.

В работе [1] экспериментально и численно исследовался теплообмен в критической точке цилиндрической модели радиусом $R_{w}=15$ мм с плоским торцом, обтекаемой дозвуковыми струями диссоциированного воздуха в различных режимах работы ВЧ-плазмотрона ВГУ-2. На рис. 8 приведены экспериментальные зависимости плотности теплового потока $q_{w}$ к охлаждаемым поверхностям различных металлов и кварца от энтальпии воздуха $\mathrm{H}_{\mathrm{e}}$ на внешней границе пограничного слоя, полученные в [1]. На основе рис. 7 и 8 по каталитиче- 
ской активности в сверхзвуковых и дозвуковых потоках диссоциированного воздуха металлы распределяются в следующем порядке: высококаталитичные - серебро, медь; среднекаталитичные - ниобий, золото, бериллий; низкокаталитичные - молибден, тантал. Кварц относится к низкокаталитичным материалам. Таким образом, наблюдается качественное соответствие между результатами настоящей работы и результатами, представленными ранее в [1]. Полученные данные по каталитичности серебра в потоке диссоциированного воздуха качественно согласуются с результатами [14] по рекомбинации атомов азота на поверхности серебра в гиперзвуковом свободномолекулярном потоке.

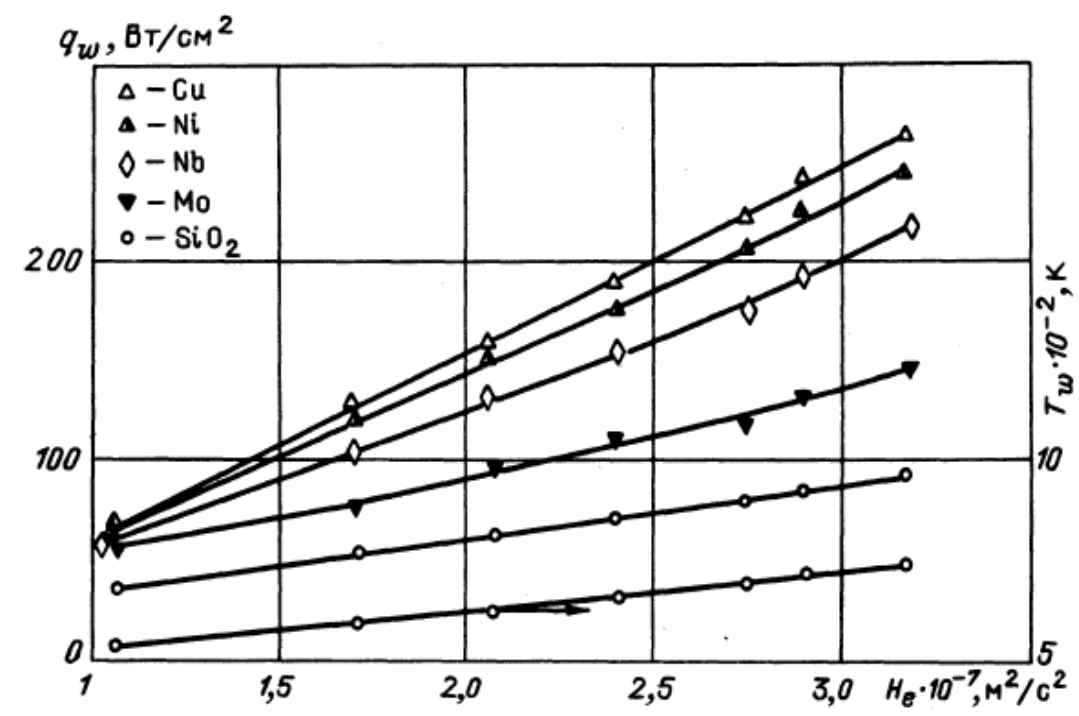

Рис. 8. Тепловые потоки к металлическим и кварцевой поверхностям и температура кварца в критической точке модели, обтекаемой дозвуковой струей диссоциированного воздуха плазмотрона ВГУ-2 в зависимости от энтальпии $\mathrm{H}_{\mathrm{e}}\left(p=100\right.$ гПа, $R_{m}=15$ мм) [1]

В работе [1] было установлено, что в дозвуковых струях диссоциированного воздуха при температуре $300 \mathrm{~K}$ медь может рассматриваться и использоваться как эталонный абсолютно каталитичный материал $(\gamma=1)$. Однако, в сверхзвуковых струях диссоциированного воздуха, согласно рис. 7, медная поверхность не может рассматриваться как абсолютно каталитичная, а соответствующее значение $\gamma=0.1$. Этот вывод экспериментально подтверждает результат [15] расчета теплового потока в критической точке с учетом образования молекул $\mathrm{N}_{2}$ и $\mathrm{O}_{2}$ в колебательно возбужденных состояниях при рекомбинации атомов $\mathrm{N}$ и О. При сверхзвуковом обтекании высококаталитичной поверхности диссоциированным газом в тонком пограничном слое не происходят процессы «тушения» колебательно возбужденных молекул и часть колебательной энергии молекулы уносят в поток [15]. При дозвуковом обтекании высококаталитичной поверхности колебательно возбужденные молекулы, образовавшиеся на поверхности в результате рекомбинации атомов, эффективно «тушатся» в относительно толстом пограничном слое. Формально это выражается в том, что при дозвуковом обтекании тепловой поток в критической точке практически не изменяется при изменении $\gamma$ в интервале $0.1 \div 1$ в отличие от сверхзвукового режима обтекания поверхности, когда имеет место сильная зависимость теплового потока от $\gamma$ для высококаталитичных поверхностей.

\section{4. Заключение}

В одном из сверхзвуковых режимов обтекания цилиндрической модели недорасширенными струями диссоциированного воздуха измерены тепловые потоки в критической точке к ряду металлов и кварца. Наибольшие тепловые потоки зарегистрированы на датчи- 
ках из серебра и меди, что свидетельствует о высокой каталитичности их поверхности, причем каталитичность серебра и меди практически одинаковы.

Вторая группа материалов - золото, бериллий и ниобий. Эти материалы оказались близки по уровню зарегистрированных тепловых потоков. Реализуемые потоки для них ниже, чем у меди и серебра. Эти металлы можно отнести к среднекаталитичным. Наименьшие тепловые потоки отмечены у молибдена и тантала.

Минимальные тепловые потоки реализованы в исследовавшемся режиме для кварцевых датчиков. Кварц может быть отнесен к низкокаталитичным материалам.

На основе численного моделирования обтекания цилиндрической модели недорасширенными струями диссоциированного воздуха рассчитаны тепловые потоки в точке торможения в зависимости от эффективного коэффициента рекомбинации атомарных компонентов $\gamma$ в диапазоне $0 \leq \gamma \leq 1$. Из сравнения экспериментальных и рассчитанных тепловых потоков определены значения $\gamma$ для перечисленных выше материалов и установлена количественная шкала каталитичности рассмотренных металлов и кварца.

Данные по каталитичности металлов и кварца качественно согласуются с результатами, полученными ранее в дозвуковых струях диссоциированного воздуха.

При обтекании сверхзвуковой струей диссоциированного воздуха охлаждаемая поверхность меди $\left(T_{w}=300 \mathrm{~K}\right)$ не может рассматриваться как эталонная абсолютно каталитичнаая поверхность по отношению к рекомбинации атомов $\mathrm{N}$ и $\mathrm{O}$, в отличие от таковой при дозвуковом обтекании.

\section{Благодарности и ссылки на гранты}

Данная работа выполнена в рамках госзадания АААА-А17-117021319383-2 при частичной поддержке гранта РФФИ №17-01-00054 с использованием Суперкомпьютерного комплекса МГУ.

\section{Литература}

1. Колесников А. Ф., Якушин М. И., Об определении эффективных вероятностей гетерогенной рекомбинации атомов по тепловым потокам к поверхности, обтекаемой диссоциированным воздухом // Математическое моделирование. - 1989. - Т. 1. - № 3. - С. 44-60.

2. Kolesnikov A. F. Combined measurements and computations of high enthalpy and plasma flows for determination of TPM surface catalycity. In: Measurement Techniques for High Enthalpy and Plasma Flows, edited by J.-M. Charbonnier and G.S.R. Sarma, RTO-EN-8, Canada, 2000.

3. Власов В.И., Залогин Г.Н., Землянский Б.А., Кнотько В.Б. Методика и результаты экспериментального определения каталитической активности материалов при высоких температурах //Известия Российской академии наук. Механика жидкости и газа. -2003. - №. 5. - С. 178-189.

4. García A., Chazot O., Fletcher D. Investigations in Plasmatron Facilities on Catalicity Determination //Fourth Symposium on Aerothermodynamics for Space Vehicles. - 2002. - T. 487. - C. 489.

5. Егоров И.В., Жестков Б.Е., Шведченко В.В. Определение каталитической активности материалов при высоких температурах в гиперзвуковой трубе ВАТ-104 // Ученые записки ЦАГИ. 2014. - T. XLV. - № 1. - С. 3-13.

6. Гордеев А.Н., Колесников А.Ф., Сахаров В.И. Течение и теплообмен в недорасширенных струях индукционного плазмотрона // Известия РАН, Механика жидкости и газа. - 2011. № 4. - С. 130-142.

7. Гордеев А.Н., Колесников А.Ф. Высокочастотные индукционные плазмотроны серии ВГУ. - В сб. Актуальные проблемы механики: Физико-химическая механика жидкостей и газов. Москва: Наука, 2010. С. 151-177.

8. Васильевский С.А., Гордеев А.Н., Колесников А.Ф., Сахаров В.И., Симоненко Е.П., Симоненко Н.П. Экспериментальное и численное исследование теплообмена высокоэнтальпийных по- 
токов воздуха с цилиндрическими моделями из меди и с образцами из керамики на ВЧплазмотроне ВГУ-4 // Физико-химическая кинетика в газовой динамике. 2018. Т. 19 (1).

http://chemphys.edu.ru/issues/2018-19-1/articles/731/

9. Afonina N.E., Gromov V.G., Sakharov V. I. HIGHTEMP technique of high temperature gas flows numerical simulations // Proc. 5th Europ. Symp. on Aerothermodyn. Spase Vehicles. Cologne, Germany, 2004. SP 563. Noordwijk: ESTEC, 2004. Pp. 323-328.

10. Сахаров В.И. Численное моделирование термическии химически неравновесных течений и теплообмена в недорасширенных струях индукционного плазмотрона // Изв. РАН. МЖГ. 2007. № 6. С. 157.

11. Термодинамические свойства индивидуальных веществ. Справочное издание. М.: Наука, 1978. Т.1. Кн. 1. 495c; Т.1. Кн. 2. 327 с.

12. Васильевский С.А., Колесников А.Ф. Численное моделирование течений равновесной индукционной плазмы в цилиндрическом канале плазмотрона // Изв. РАН. МЖГ. - 2000. - № 5. C. $164-173$.

13. Гордеев А.Н., Колесников А.Ф., Сахаров В.И. Экспериментальное и численное исследование теплообмена высокоэнтальпийных недорасширенных струй воздуха с цилиндрическими моделями // Изв. РАН. МЖГ. 2018. №5. С. 125-133:

14. Жестков Б.Е., Книвель А.Я. Взаимодействие диссоциированного потока азота с металлическими поверхностями // Ученые записки ЦАГИ. - 1979. - Т. Х. - № 6. - С. 37-46.

15. Беркут В.Д., Дорошенко В.М., Ковтун В.В., Кудрявцев Н.Н. Неравновесные физикохимические процессы в гиперзвуковой аэродинамике. М.: Энергоатомиздат. 1994. 400 с.

Статья поступила в редакцию 23 января 2019 г. 